\title{
Reynolds Turbulence Solution
}

\author{
Bohua $\mathrm{SUN}^{1}$ \\ ${ }^{1}$ School of Civil Engineering 8 Institute of Mechanics and Technology, \\ Xi'an University of Architecture and Technology, Xi'an 710055, China \\ http://imt.xauat.edu.cn \\ email: sunbohua@xauat.edu.cn
}

(Dated: August 6, 2019)

\begin{abstract}
The study found an error in current literature, including textbooks, about the number of unknowns in the Reynolds stress tensor and/or in Reynolds-averaged Navier-Stokes equations (RANS). Current literature claims that the Reynolds stress tensor has six unknowns; however, this article shows that the Reynolds stress tensor only has three unknowns, namely the three components of fluctuation velocity. This research discovers that the misconception about the number of unknowns in the RANS could stem from misinterpreting the Reynolds stress tensor. The misconception might be one of the biggest scientific mistake in classical physics and has hampered the development of turbulence for longtime. In order to find a way out of this difficult situation, we return to the time of Reynolds in 1895 and revisit Reynolds' averaging formulation of turbulence. In light of Reynolds' deterministic view on turbulence, this paper proposes a general algorithm for three dimensional turbulence flows. The study found that the magnitude of velocity fluctuations or turbulence is proportional to the flow pressure, which is a remarkable discovery. As applications, the Reynolds turbulence solution of the turbulent Burgers equation and the Prandtl boundary layer equations have been obtained, the beauty of these relevant solutions is that there is no adjustable parameters. The present investigation can be considered as a renaissance of Reynolds' work in 1895, which might shed light on the wellknown closure problem of turbulence, and help to understand the puzzle of the turbulence closure problem that has eluded scientists and mathematicians for centuries.
\end{abstract}

Keywords: Turbulence, number of unknowns, the Reynolds stress tensor, RANS, turbulence closure problem

\section{INTRODUCTION}

You're on a airplane when you feel a sudden jolt. Outside your window nothing seems to be happening, yet the plane continues to rattle you and your fellow passenger$\mathrm{s}$ as it passes through turbulent air in the atmosphere. And yet, turbulence is ubiquitous, springing up in virtually any system that has moving fluids. That includes that airflow in your respiratory tract. The blood moving through your arteries. And the coffee in your cup as you stir it. Clouds are governed by turbulence, as are waves crashing along the shore and the gusts of plasma in our Sun. Understanding precisely how this phenomenon works would have a bearing on so many aspects of our lives $[1-60]$.

The turbulence phenomenon is one of the greatest prevailing unsolved mysteries of physics. After more than a century of studying turbulence, we've found a few answers on how it works, and affects the world around us. Most scientists argue that this will be achieved relying on statistics and increased computing power. Extremely high-speed computer simulations of turbulent flows may help to identify patterns that lead to a theory that organizes and unifies predictions for different situations. Other scientists state that the phenomenon is so complex that such a full-fledged theory will not be possible [1-60].

In respect of the turbulence problem, a myriad of tentative theories have been proposed and each with its own doctrines and beliefs, whilst often focused on particular experiments; however, there is not much in the way of a coherent theoretical framework [5, 7-16, 37-39]. Turbulence is a unique subject, which engineers, mathematicians and physicists view in differently [34]. Many engineers promote the use of semi-empirical models of turbulence [34], while mathematicians advocate the use of purely statistical models [4, 6, 28, 29, 42-45, 51, 52], the renomalization model [32], and the formalism of chaos theory and fractals [46-48].

In 1972 a new chapter was launched in turbulence theory: [49] demonstrated that it was possible to perform direct numerical simulation (DNS) of a fully turbulent flow. It is important to understand that DNS does not require any turbulence model to parameterize influence of the turbulent eddies. Rather, every eddy, from the largest to the smallest, is computed. From a technical perspective, the turbulence can be solved by DNS if computers have infinite speed. However, a huge chasm remains between what the engineer needs to know, and what can be realized by DNS, using current computers. Even if DNS can assist to solve turbulence issues and problems, one still requires turbulence modelling to acquire a physical understanding of it [35].

Although the above-mentioned professionals have different views about turbulence, there is consensus that the deterministic Navier-Stokes equation probably contain$\mathrm{s}$ all the information about turbulence [5]. Turbulence prediction can be attained by understanding solutions for the Navier-Stokes equations [4, 5, 7-24, 26-60]. Navier [20] and Poisson [21] first obtained these equations, which were finally shown by St. Venant and Sir Gabbriel Stokes $[22,23]$ on various considerations as to the mutual action 
of the ultimate molecules of fluids [18, 19]. Hence, numerous works have been reported on various aspects of the Navier-Stokes equation. [5, 7-18, 24, 27, 37-39].

The article adopts Reynolds's deterministic view on the turbulence [18], and revisits the Reynolds-averaged Navier-Stokes equations (RANS). This article deals with a basic problem in turbulence analysis, namely the number of unknowns in the Reynolds stress tensor. This is obviously a fundamental question in fluid mechanics as well. The research in this paper reveals that the RANS only has three unknowns instead of six, as shown in current literature, including textbooks [7-14, 17, 28-31, 3739].

Although the Reynolds-averaged-Navier-StokesEquations (RANS) have been formulated for more than 120 years, all current literature and standard turbulence textbooks have incorrect information about the number of unknowns and this prevented solving the turbulence problem. Hence, if the number of unknowns are incorrect, no solutions will be possible. This situation must be corrected immediately, else it will be harmful for turbulence studies.

In view of the fact that no essential result on turbulence definition has been made, it would probably be fitting to return to the beginning of turbulent research, that is, in 1895, to study Reynolds' seminal studies to rediscover certain useful information.

The aims of this paper is to revisit Reynolds' averaging formulation of turbulence, and to clarify the number of unknowns in the Reynolds stress tensor and/or in RANS. The paper is organized as follows: following an introduction, we introduce Reynolds velocity decomposition and reformulate Reynolds-averaged Navier-Stokes equations in tensorial format; we point out an important question about the number of unknowns in the RANS; we provide three mathematical lemma and evidence about the number of unknowns; and propose approximated solutions for two problems. Finally, the paper concludes with perspectives about the future development of turbulence research studies.

\section{REYNOLDS-AVERAGED NAVIER-STOKES EQUATIONS AND DETERMINISTIC NATURE}

In ground-breaking research, Reynolds [18], considered turbulence from a different perspective. Assuming that turbulent motion already exists, he sought to establish a criterion, which decides whether the turbulent character will increase or diminish, or remain stationary [19].

In 1895 Reynolds proposed that flow velocity $\boldsymbol{u}$ and pressure $p$ are decomposed into its time-averaged quantities, $\overline{\boldsymbol{u}}, \bar{p}$, and fluctuating quantities, $\boldsymbol{u}^{\prime}, p^{\prime}$; thus, the Reynolds' decompositions are:

$$
\begin{aligned}
\boldsymbol{u} & =\overline{\boldsymbol{u}}(\boldsymbol{x})+\boldsymbol{u}^{\prime}(\boldsymbol{x}, t), \\
p(\boldsymbol{x}, t) & =\bar{p}(\boldsymbol{x})+p^{\prime}(\boldsymbol{x}, t),
\end{aligned}
$$

where coordinates and time are $(\boldsymbol{x}, t)$. According to
Reynolds, $\overline{\boldsymbol{u}}$ represent a mean-motion at each point and $\boldsymbol{u}^{\prime}$ a motion at the same point as the mean-motion at the point. Therefore, Reynolds called the $\overline{\boldsymbol{u}}$ mean-motion and $\boldsymbol{u}^{\prime}$ relative-mean-motion [18]. Both velocity and velocity and pressure time-averaging definitions are defined as

$$
\begin{aligned}
& \overline{\boldsymbol{u}}(\boldsymbol{x})=\lim _{T \rightarrow \infty} \frac{1}{T} \int_{t_{0}}^{t_{0}+T} \boldsymbol{u}(\boldsymbol{x}, t) d t \\
& \bar{p}(\boldsymbol{x})=\lim _{T \rightarrow \infty} \frac{1}{T} \int_{t_{0}}^{t_{0}+T} p(\boldsymbol{x}, t) d t
\end{aligned}
$$

where $T$ is the period of time when the averaging takes place and must be sufficiently large to give meaningful averages to measure mean values, depending on the accuracy desired. Naturally, the time-fluctuation velocity $\boldsymbol{u}^{\prime}=\boldsymbol{u}-\overline{\boldsymbol{u}}(\boldsymbol{x})$, pressure $p^{\prime}=p-\bar{p}(\boldsymbol{x})$; and their timeaveraging vanish, namely $\overline{\boldsymbol{u}}^{\prime}=\mathbf{0}$ and $\overline{p^{\prime}}=0$.

Decomposition causes the Navier-Stokes equation to transform into Reynolds-averaged Navier-Stokes equations (RANS), as follows

$$
\begin{aligned}
\rho \nabla \cdot(\overline{\boldsymbol{u}} \otimes \overline{\boldsymbol{u}})+\nabla \bar{p} & =\mu \nabla^{2} \overline{\boldsymbol{u}}+\nabla \cdot \boldsymbol{\tau}, \\
\nabla \cdot \overline{\boldsymbol{u}} & =0
\end{aligned}
$$

where dynamic viscosity $\mu$, gradient operator $\nabla=\boldsymbol{e}_{i} \nabla_{i}$, base vector in the i-coordinate $\boldsymbol{e}_{i}$, and tensor product $\otimes$, and the Reynolds stress tensor is given by

$$
\boldsymbol{\tau}=-\rho \overline{\boldsymbol{u}^{\prime} \otimes \boldsymbol{u}^{\prime}}=-\rho \lim _{T \rightarrow \infty} \frac{1}{T} \int_{t_{0}}^{t_{0}+T}\left(\boldsymbol{u}^{\prime} \otimes \boldsymbol{u}^{\prime}\right) d t,
$$

which reveals that Reynolds stress is apparent, depending on the fluctuating velocity field $\boldsymbol{u}^{\prime}$.

Denoting kinematic viscosity $\nu=\mu / \rho$, the above equations can be equivalently rewritten in conventional form, namely

$$
\begin{aligned}
\overline{\boldsymbol{u}} \cdot \boldsymbol{\nabla} \overline{\boldsymbol{u}}+\frac{1}{\rho} \boldsymbol{\nabla} \bar{p} & =\nu \boldsymbol{\nabla}^{2} \overline{\boldsymbol{u}} \\
& -\lim _{T \rightarrow \infty} \frac{1}{T} \int_{t_{0}}^{t_{0}+T}\left(\boldsymbol{u}^{\prime} \cdot \boldsymbol{\nabla} \boldsymbol{u}^{\prime}\right) d t \\
\boldsymbol{\nabla} \cdot \overline{\boldsymbol{u}} & =0
\end{aligned}
$$

These integral-differential equations are called Reynoldsaveraged Navier-Stokes equations (RANS), which were the equations (15) in [18] that Reynolds formulated, calling them equations of mean-motion at every point. It is clear that the RANS are deterministic equations rather than statistical ones [18, 19].

It is worth pointing out that for the deterministic velocity field $\boldsymbol{u}(\boldsymbol{x}, t)$, the Reynolds decomposition in Eqs. 1 and 2 can always be well-defined by Eqs. 3 and 4 . In other words, Reynolds decomposition can always be constructed for any flow motion, regardless of turbulent flow or not.

It is misleading to consider the essence of turbulence as a random motion, and the flow velocity field $\boldsymbol{u}$ as a 
statistical function [28]. Because there is consensus on turbulence, namely that the deterministic Navier-Stokes equations contain all the required information about turbulence [5], means that the essence of the flow velocity and its Navier-Stokes equations is deterministic, rather than random. Taylor [28] introduced a statistical approach and attempted to study turbulence from a statistical point of view; however, the approach is merely a mathematical method for solutions, which cannot change the deterministic nature of flow velocity $\boldsymbol{u}$ and NavierStokes equations. In physics, turbulence cannot be considered as a random motion simply because it is difficulty to solve. Generally speaking, any mathematical method used for solving a problem should not change the physics of the problem.

\section{HOW MANY UNKNOWNS ARE THERE IN THE REYNOLDS STRESS TENSOR AND/OR REYNOLDS-AVERAGED NAVIER-STOKES EQUATIONS ?}

All current literature including textbooks, report that the Reynolds stresses $\boldsymbol{\tau}$ have six unknowns (Later we will show that the Reynolds stresses only have three unknowns instead of six). This traditional understanding has resulted in consensus that there are 10 unknowns as shown in Eqs.5, 6 and/or Eqs.36, 37.

In his 1895 paper [18], Reynolds did not discuss the number of unknowns in the RANS. However, from his presentation we note that he never considered the term $\rho \overline{\boldsymbol{u}^{\prime} \otimes \boldsymbol{u}^{\prime}}$ as independent unknowns, while for some cases he proposed explicit expressions for the velocity fluctuation $\boldsymbol{u}^{\prime}$ on page 149 , and equation (50) on page 158 of his paper [18]. For instance, one expression he proposed on page 149 of [18] is:

$$
\sum\left[A_{r} \cos \left\{r\left(n t+\frac{2 \pi}{a} x\right)\right\}\right]
$$

and in the section, Expressions for the components of possible relative-mean-motion, he proposed a similar expression on page 15 of [18], as follows:

$$
\begin{aligned}
& u^{\prime}=\sum_{0}^{\infty}\left\{\left(\frac{d \alpha_{n}}{d y}+\frac{d \gamma_{n}}{d z} \cos (n l x)+\left(\frac{d \beta_{n}}{d y}+\frac{d \delta_{n}}{d z}\right) \sin (n l x)\right\},\right. \\
& v^{\prime}=\sum_{0}^{\infty}\left\{n \alpha_{n} \sin (n l x)-n l \beta_{n} \cos (n l x)\right\}, \\
& w^{\prime}=\sum_{0}^{\infty}\left\{n \gamma_{n} \sin (n l x)-n l \delta_{n} \cos (n l x)\right\} .
\end{aligned}
$$

Reynolds also compiled integrations by using the above expressions, for example, equation (58) on page 159 of
Reynolds's research [18], which is shown below:

$$
\begin{aligned}
& \iint \rho \overline{u^{\prime} v^{\prime}} \frac{d \bar{u}}{d y} d y d z \\
& =\frac{1}{2} \iint \sum\left[n l\left(\alpha_{n} \frac{d \beta_{n}}{d y}-\beta_{n} \frac{d \alpha_{n}}{d y}\right) \frac{d \bar{u}}{d y}\right] d y d z .
\end{aligned}
$$

Given the above expressions means that Reynolds regarded the velocity fluctuation $\boldsymbol{u}^{\prime}$ not only as unknowns but also deterministic quantity (not a random one !). Please see Reynolds research paper [18] for the meaning of the notations in the above expressions..

Due to limited references, the author does not know who was the first person who proposed the 10-unknowns perception in the RANS. This mistake in classical physics has hampered the development of turbulence.

The RANS in equation 5 and 6 have four independent equations that governs the mean velocity field, namely the three components of the Reynolds equation; equation 5 together with the mean continuity equation 6. However, these four equations contain more than four unknowns. In addition to $\overline{\boldsymbol{u}}$ and $\bar{p}$, there are also the Reynolds stresses $\boldsymbol{\tau}$, which results in the Reynoldsaveraged Navier-Stokes equations being unclosed. The closure problem has been considered as the number one topic in turbulence during the past several decades, while scientists and engineers have made many attempts to solve this closure problem, however, no universal modelling has been proposed.

Regarding the RANS closure problem, the curren$\mathrm{t}$ consensus is that there are six unknown components in the symmetric Reynolds stress tensor $\boldsymbol{\tau}$, namely $\tau_{11}, \tau_{12}, \tau_{13}, \tau_{22}, \tau_{23}, \tau_{33}$. However, this research study presents a complete different perspectives. The Reynold$\mathrm{s}$ stress tensor $\boldsymbol{\tau}$ only has three unknowns, namely the velocity fluctuation components $u_{i}^{\prime}(i=1,2,3)$ owing to the fact that the Reynolds stress tensor is simply an integration of a second order dyadic tensor of flow velocity fluctuations rather than a general symmetric tensor.

\section{LEMMA}

Following lemmas support the above statement.

Lemma 1 Given two vectors, $\boldsymbol{v}(\boldsymbol{x}, t)=v_{i} \boldsymbol{e}_{i}=v_{1} \boldsymbol{e}_{1}+$ $v_{2} \boldsymbol{e}_{2}+v_{3} \boldsymbol{e}_{3}$ and $\boldsymbol{w}(\boldsymbol{x}, t)=w_{j} \boldsymbol{e}_{j}=w_{1} \boldsymbol{e}_{1}+w_{2} \boldsymbol{e}_{2}+w_{3} \boldsymbol{e}_{3}$, we can define a dyadic tensor $\boldsymbol{v} \otimes \boldsymbol{w}$ as follows [61]

$$
\begin{aligned}
\boldsymbol{v} \otimes \boldsymbol{w} & =v_{i} \boldsymbol{e}_{i} \otimes w_{j} \boldsymbol{e}_{j}=v_{i} w_{j} \boldsymbol{e}_{i} \otimes \boldsymbol{e}_{j} \\
& =v_{1} w_{1} \boldsymbol{e}_{1} \otimes \boldsymbol{e}_{1}+v_{1} w_{2} \boldsymbol{e}_{1} \otimes \boldsymbol{e}_{2}+v_{1} w_{3} \boldsymbol{e}_{1} \otimes \boldsymbol{e}_{3} \\
& +v_{2} w_{1} \boldsymbol{e}_{2} \otimes \boldsymbol{e}_{1}+v_{2} w_{2} \boldsymbol{e}_{2} \otimes \boldsymbol{e}_{2}+v_{2} w_{3} \boldsymbol{e}_{2} \otimes \boldsymbol{e}_{3} \\
& +v_{3} w_{1} \boldsymbol{e}_{3} \otimes \boldsymbol{e}_{1}+v_{3} w_{2} \boldsymbol{e}_{3} \otimes \boldsymbol{e}_{2}+v_{3} w_{3} \boldsymbol{e}_{3} \otimes \boldsymbol{e}_{3}
\end{aligned}
$$

In general, in the case of $\boldsymbol{v} \neq \boldsymbol{w}$, the dyadic tensor $\boldsymbol{v} \otimes \boldsymbol{w}$ is a general tensor with 9 components (elements) and has 6 unknowns, namely $v_{1}, v_{2}$ and $v_{3}$ and $w_{1}, w_{2}$ 
and $w_{3}$, since the 9 components can be fully determined by the 6 unknowns.

If $\boldsymbol{v}=\boldsymbol{w}$, the tensor $\boldsymbol{v} \otimes \boldsymbol{w}$ is a 2rd order tensor with 9 components (elements), and have 3 unknowns, namely $v_{1}, v_{2}$ and $v_{3}$ and/or $w_{1}, w_{2}$ and $w_{3}$, since the 9 components can be fully determined by the 3 unknowns.

Lemma 2 Given three vectors, $\boldsymbol{u}(\boldsymbol{x}, t)=u_{i} \boldsymbol{e}_{i}=u_{1} \boldsymbol{e}_{1}+$ $u_{2} \boldsymbol{e}_{2}+u_{3} \boldsymbol{e}_{3}, \boldsymbol{v}(\boldsymbol{x}, t)=v_{i} \boldsymbol{e}_{i}=v_{1} \boldsymbol{e}_{1}+v_{2} \boldsymbol{e}_{2}+v_{3} \boldsymbol{e}_{3}$ and $\boldsymbol{w}(\boldsymbol{x}, t)=w_{j} \boldsymbol{e}_{j}=w_{1} \boldsymbol{e}_{1}+w_{2} \boldsymbol{e}_{2}+w_{3} \boldsymbol{e}_{3}$, we can define $a$ 3rd order tensor $\boldsymbol{v} \otimes \boldsymbol{w}$ as follows

$\boldsymbol{u} \otimes \boldsymbol{v} \otimes \boldsymbol{w}=u_{i} \boldsymbol{e}_{k} \otimes v_{k} \boldsymbol{e}_{i} \otimes w_{j} \boldsymbol{e}_{j}=u_{k} v_{i} w_{j} \boldsymbol{e}_{k} \otimes \boldsymbol{e}_{i} \otimes \boldsymbol{e}_{j}$

In general, in the case of $\boldsymbol{u} \neq \boldsymbol{v} \neq \boldsymbol{w}$, the tensor $\boldsymbol{u} \otimes$ $\boldsymbol{v} \otimes \boldsymbol{w}$ is a general tensor with 27 components (elements) and has 9 unknowns, namely $u_{1}, u_{2}$ and $u_{3}, v_{1}, v_{2}$ and $v_{3}$ and $w_{1}, w_{2}$ and $w_{3}$, since the 27 components can be fully determined by the 9 unknowns.

If $\boldsymbol{u}=\boldsymbol{v}=\boldsymbol{w}$, the tensor $\boldsymbol{u} \otimes \boldsymbol{v} \otimes \boldsymbol{w}$ is a 3rd order tensor with 27 components (elements) and has 3 unknowns, namely $u_{1}, u_{2}$ and $u_{3}$, or $v_{1}, v_{2}$ and $v_{3}$ and/or $w_{1}, w_{2}$ and $w_{3}$, since the 27 components can be fully determined by the 3 unknowns.

Lemma 3 Given a vector, $\boldsymbol{v}(\boldsymbol{x}, t)=v_{i} \boldsymbol{e}_{i}=v_{1} \boldsymbol{e}_{1}+v_{2} \boldsymbol{e}_{2}+$ $v_{3} \boldsymbol{e}_{3}$, we can define a 2nd order symmetric dyadic tensor $\boldsymbol{v} \otimes \boldsymbol{v}$ and its mean value $\boldsymbol{A}(\boldsymbol{x})$ as follows

$$
\begin{aligned}
\boldsymbol{A}(\boldsymbol{x}) & =\lim _{T \rightarrow \infty} \frac{1}{T} \int_{t_{0}}^{t_{0}+T} \boldsymbol{v} \otimes \boldsymbol{v} d t \\
& =\lim _{T \rightarrow \infty} \frac{1}{T} \int_{t_{0}}^{t_{0}+T} v_{i} v_{j} \boldsymbol{e}_{i} \otimes \boldsymbol{e}_{j} d t \\
& =\lim _{T \rightarrow \infty} \frac{1}{T} \int_{t_{0}}^{t_{0}+T}\left[v_{1} v_{1} \boldsymbol{e}_{1} \otimes \boldsymbol{e}_{1}+v_{1} v_{2} \boldsymbol{e}_{1} \otimes \boldsymbol{e}_{2}\right. \\
& +v_{1} v_{3} \boldsymbol{e}_{1} \otimes \boldsymbol{e}_{3}+v_{2} v_{1} \boldsymbol{e}_{2} \otimes \boldsymbol{e}_{1}+v_{2} v_{2} \boldsymbol{e}_{2} \otimes \boldsymbol{e}_{2} \\
& +v_{2} v_{3} \boldsymbol{e}_{2} \otimes \boldsymbol{e}_{3}+v_{3} v_{1} \boldsymbol{e}_{3} \otimes \boldsymbol{e}_{1} \\
& \left.+v_{3} v_{2} \boldsymbol{e}_{3} \otimes \boldsymbol{e}_{2}+v_{3} v_{3} \boldsymbol{e}_{3} \otimes \boldsymbol{e}_{3}\right] d t
\end{aligned}
$$

where $v_{i} v_{j}=v_{j} v_{i}$.

Although $\boldsymbol{A}(\boldsymbol{x})$ has six independent components, namely $v_{1} v_{1}, v_{1} v_{2}, v_{1} v_{3}, v_{2} v_{2}, v_{2} v_{3}, v_{3} v_{3}$, it is clear that there are only three independent quantities, namely $v_{1}, v_{2}$ and $v_{3}$, in the $\boldsymbol{A}(\boldsymbol{x})$. It is because the quantities $v_{1} v_{1}, v_{1} v_{2}, v_{1} v_{3}, v_{2} v_{2}, v_{2} v_{3}, v_{3} v_{3}$ can be fully determined by $v_{1}, v_{2}$ and $v_{3}$.

Lemma 3 actually states that any (time) averaging operation is merely a method of data processing and will not change the number of unknowns within the problem.

It is clear that $A(\boldsymbol{x})$ will be the Reynolds stress tensor $\tau$ if replacing $v_{1}, v_{2}$ and $v_{3}$ by the components of velocity fluctuations $u_{i}^{\prime}(i=1,2,3)$, respectively, namely $v_{1}=$ $u_{1}^{\prime}, v_{2}=u_{2}^{\prime}, v_{3}=u_{3}^{\prime}$.

\section{THREE PROOFS}

The number of unknowns in the Reynolds stress tensor and/or in the RANS should not been a issue at all, since you can simply find it from Reynolds' papers [18]. However, due to the long-time misconception in turbulence research community, it has affected people's mind. To make it clear, it is necessary to explain the issue from the following aspects.

\section{A. Direct proof by the definition of Reynolds stress tensor}

The Lemma shows that the Reynolds stress tensor olny has three unknown components. This is proved when the Reynolds stress tensor is defined in the following

$$
\begin{aligned}
\boldsymbol{\tau} & =-\rho \overline{\boldsymbol{u}^{\prime} \otimes \boldsymbol{u}^{\prime}}=-\rho \overline{u_{i}^{\prime} \boldsymbol{e}_{i} \otimes u_{j}^{\prime} \boldsymbol{e}_{j}} \\
& =-\rho \overline{u_{i}^{\prime} u_{j}^{\prime}} \boldsymbol{e}_{i} \otimes \boldsymbol{e}_{j} \\
& =-\rho \lim _{T \rightarrow \infty} \frac{1}{T} \int_{t_{0}}^{t_{0}+T}\left(u_{i}^{\prime} u_{j}^{\prime} \boldsymbol{e}_{i} \otimes \boldsymbol{e}_{j}\right) d t \\
& =\left[-\rho \lim _{T \rightarrow \infty} \frac{1}{T} \int_{t_{0}}^{t_{0}+T} u_{i}^{\prime} u_{j}^{\prime} d t\right] \boldsymbol{e}_{i} \otimes \boldsymbol{e}_{j} \\
& =\tau_{i j} \boldsymbol{e}_{i} \otimes \boldsymbol{e}_{j},
\end{aligned}
$$

and the fluctuation velocity convective terms are:

$$
\begin{aligned}
& \overline{\boldsymbol{u}^{\prime} \cdot \boldsymbol{\nabla} \boldsymbol{u}^{\prime}}=\overline{u_{i}^{\prime} \boldsymbol{e}_{i} \cdot\left[\boldsymbol{e}_{k} \partial_{k} \otimes\left(u_{j}^{\prime} \boldsymbol{e}_{j}\right)\right]} \\
& =\overline{u_{i}^{\prime} u_{j, k}^{\prime}} \boldsymbol{e}_{i} \cdot\left(\boldsymbol{e}_{k} \otimes \boldsymbol{e}_{j}\right)=\overline{u_{i}^{\prime} u_{j, k}^{\prime}}\left(\boldsymbol{e}_{i} \cdot \boldsymbol{e}_{k}\right) \boldsymbol{e}_{j} \\
& =\overline{u_{i}^{\prime} u_{j, k}^{\prime}} \delta_{i k} \boldsymbol{e}_{j}=\overline{u_{i}^{\prime} u_{j, i}^{\prime}} \boldsymbol{e}_{j} \\
& =\left(\lim _{T \rightarrow \infty} \frac{1}{T} \int_{t}^{t+T} u_{i}^{\prime} u_{j, i}^{\prime} d t\right) \boldsymbol{e}_{j} \\
& =\tau_{i j, i} \boldsymbol{e}_{j}=\nabla \cdot \tau,
\end{aligned}
$$

where the Reynolds stress tensor in index format $\tau_{i j}$ is defined by the following

$$
\tau_{i j}=\tau_{j i}=-\rho \lim _{T \rightarrow \infty} \frac{1}{T} \int_{t_{0}}^{t_{0}+T} u_{i}^{\prime} u_{j}^{\prime} d t .
$$

The above indicates that any Reynolds stress tensor component $\tau_{i j}$ can be calculated by fluctuation velocity components $u_{1}^{\prime}, u_{2}^{\prime}$ and $u_{3}^{\prime}$, which means that the $\tau_{i j}$ is dependent on components $u_{1}^{\prime}, u_{2}^{\prime}$ and $u_{3}^{\prime}$. In other words, components $u_{1}^{\prime}, u_{2}^{\prime}$ and $u_{3}^{\prime}$ are real unknowns. This is the main reason why stating that that the Reynolds stress tensor $\boldsymbol{\tau}$ has 6 unknowns could be the biggest error in the history of turbulence modelling.

Therefore, the formulation in equation 13 reveals that the Reynolds stress tensor $\boldsymbol{\tau}=-\rho \overline{\boldsymbol{u}^{\prime} \otimes \boldsymbol{u}^{\prime}}$ can be fully calculated by three independent components of fluctuation velocity, namely $u_{1}^{\prime}, u_{2}^{\prime}$ and $u_{3}^{\prime}$. In other words, the Reynolds stress tensor only has three unknowns rather 
than six. It means that the averaging technique is merely a mathematical process, which can provide a mean value, but cannot change the number of unknowns within the problem.

The misinterpretation of the number of unknown components in the literature may stem from considering the Reynolds stress tensor as a general 2nd order symmetric tensor with six independent components. However, the Reynolds stress tensor is not an arbitrary 2nd order tensor. In fact, its components are made by the bi-product of the fluctuation velocity components, which means that the Reynolds stress tensor is a dyadic tensor of the velocity fluctuation. The unknown components to construct the dyadic tensor are the three components of fluctuation velocity $\boldsymbol{u}^{\prime}$. Therefore, the Reynolds stress tensor only has three unknowns, namely $u_{1}^{\prime}, u_{2}^{\prime}$ and $u_{3}^{\prime}$. For two dimensional flow, of course, the 2D Reynolds stress tensor only has two unknowns, namely $u_{1}^{\prime}, u_{2}^{\prime}$.

\section{B. Proof of a particular case}

One can use two scalar functions, $u^{\prime}=\sqrt{2} U \cos (\omega t)$ and $v^{\prime}=\sqrt{2} V \cos (\omega t+\theta)$, as independent unknowns, to construct a 2nd order tensor or matrix with 4 components, as follows: $\left(u^{\prime}\right)^{2}, u^{\prime} v^{\prime}, v^{\prime} u^{\prime},\left(v^{\prime}\right)^{2}$. Thus, the time averages are:

$$
\begin{aligned}
\lim _{T \rightarrow \infty} \frac{1}{T} \int_{t_{0}}^{t_{0}+T}\left(u^{\prime} u^{\prime}\right) d t & =U^{2} \\
\lim _{T \rightarrow \infty} \frac{1}{T} \int_{t_{0}}^{t_{0}+T}\left(u^{\prime} v^{\prime}\right) d t & =U V \cos \theta \\
\lim _{T \rightarrow \infty} \frac{1}{T} \int_{t_{0}}^{t_{0}+T}\left(v^{\prime} u^{\prime}\right) d & =U V \cos \theta \\
\lim _{T \rightarrow \infty} \frac{1}{T} \int_{t_{0}}^{t_{0}+T}\left(v^{\prime} v^{\prime}\right) d t & =V^{2}
\end{aligned}
$$

In the definition of the Reynolds stress tensor, $\theta=0$, we have $u^{\prime}=\sqrt{2} U \cos (\omega t)$ and $v^{\prime}=\sqrt{2} V \cos (\omega t)$ and, the averaging are as follows

$$
\begin{aligned}
& \lim _{T \rightarrow \infty} \frac{1}{T} \int_{t_{0}}^{t_{0}+T}\left(u^{\prime} u^{\prime}\right) d t=U^{2}, \\
& \lim _{T \rightarrow \infty} \frac{1}{T} \int_{t_{0}}^{t_{0}+T}\left(u^{\prime} v^{\prime}\right) d t=U V, \\
& \lim _{T \rightarrow \infty} \frac{1}{T} \int_{t_{0}}^{t_{0}+T}\left(v^{\prime} u^{\prime}\right) d t=U V, \\
& \lim _{T \rightarrow \infty} \frac{1}{T} \int_{t_{0}}^{t_{0}+T}\left(v^{\prime} v^{\prime}\right) d t=V^{2} .
\end{aligned}
$$

Therefore, the Reynolds stress tensor is

$$
\begin{gathered}
\boldsymbol{\tau}=-\rho\left(U^{2} \boldsymbol{e}_{1} \otimes \boldsymbol{e}_{1}+U V \boldsymbol{e}_{1} \otimes \boldsymbol{e}_{2}\right. \\
\left.+V U \boldsymbol{e}_{2} \otimes \boldsymbol{e}_{1}+V^{2} \boldsymbol{e}_{2} \otimes \boldsymbol{e}_{2}\right)
\end{gathered}
$$

The above process shows that, $u^{\prime}$ or $U$, and $v^{\prime}$ or $V$, are the independent unknowns, which culminate in the Reynolds stress tensor $\boldsymbol{\tau}$.

If the $\theta$ remains, then we can refer to the time correlation (autocorrelations) at the same point. The correlation between the same (Greek autos = self or same) fluctuating quantity measured at two different times (at the same point in space) is not relevant to the behaviour of turbulence, while its measurement requires a time delay mechanism (usually a tape recorder with movable heads or a digital sample-and-delay system).

In the same way, one can define the space correlation; however, all the literature, including textbooks, mention that the Reynolds stress tensor is the one-point or singlepoint velocity fluctuation correlation. Therefore, $\theta=0$.

Even without $\theta=0$, the Reynolds stress still has three independent unknowns, namely the three components of the velocity fluctuations.

\section{Proof of a symmetric tensor spectral representation}

To support this obvious statement from another point of view, the following considered the tensor representation theory by presenting the Reynolds stress tensor as follows:

$$
\boldsymbol{\tau}=-\rho \lim _{T \rightarrow \infty} \frac{1}{T} \int_{t_{0}}^{t_{0}+T} \boldsymbol{\sigma} d t
$$

where $\boldsymbol{\sigma}=\boldsymbol{u}^{\prime}(\boldsymbol{x}, t) \otimes \boldsymbol{u}^{\prime}(\boldsymbol{x}, t)=u_{i}^{\prime} \boldsymbol{e}_{i} \otimes u_{j}^{\prime} \boldsymbol{e}_{j}=u_{i}^{\prime} u_{j}^{\prime} \boldsymbol{e}_{i} \otimes \boldsymbol{e}_{j}$.

The characteristic equation of $\boldsymbol{\sigma}$ is

$$
\lambda^{3}-I_{1} \lambda^{2}+I_{2} \lambda-I_{3}=0 .
$$

Note $\operatorname{tr}(\boldsymbol{\sigma})^{2}=\left(u_{1}^{\prime}\right)^{2}+\left(u_{2}^{\prime}\right)^{2}+\left(u_{3}^{\prime}\right)^{2}$ and $\operatorname{tr}(\boldsymbol{\sigma})^{2}=\operatorname{tr}\left(\boldsymbol{\sigma}^{2}\right)$, thus

$$
\begin{aligned}
I_{1} & =\operatorname{tr}(\boldsymbol{\sigma})=\left(u_{1}^{\prime}\right)^{2}+\left(u_{2}^{\prime}\right)^{2}+\left(u_{3}^{\prime}\right)^{2} \\
I_{2} & =I_{2}=\frac{1}{2}\left[\left(\operatorname{tr}(\boldsymbol{\sigma})^{2}-\operatorname{tr}\left(\boldsymbol{\sigma}^{2}\right)\right]=0\right. \\
I_{3} & =\operatorname{det}(\boldsymbol{\sigma})=\varepsilon_{i j k}\left(u_{1} u_{j}\right)\left(u_{2} u_{j}\right)\left(u_{3} u_{j}\right) \\
& =\left(u_{1}^{\prime} u_{2}^{\prime} u_{3}^{\prime}\right)^{2}-\left(u_{1}^{\prime} u_{2}^{\prime} u_{3}^{\prime}\right)^{2}-\left(u_{1}^{\prime} u_{2}^{\prime} u_{3}^{\prime}\right)^{2} \\
& +\left(u_{1}^{\prime} u_{2}^{\prime} u_{3}^{\prime}\right)^{2}+\left(u_{1}^{\prime} u_{2}^{\prime} u_{3}^{\prime}\right)^{2}-\left(u_{1}^{\prime} u_{2}^{\prime} u_{3}^{\prime}\right)^{2} \\
& =0
\end{aligned}
$$

Therefore, the characteristic equation of $\boldsymbol{\sigma}$ in Eq.25 has three roots, as follows

$$
\lambda_{1}=0, \lambda_{2}=I_{1}, \lambda_{3}=0
$$

From the spectra representation theory of a symmetric tensor, we can rewrite the tensor $\boldsymbol{\sigma}$, as follows

$$
\begin{aligned}
\boldsymbol{\sigma} & =\lambda_{1} \hat{\boldsymbol{e}}_{1} \otimes \hat{\boldsymbol{e}}_{1}+\lambda_{2} \hat{\boldsymbol{e}}_{2} \otimes \hat{\boldsymbol{e}}_{2}+\lambda_{3} \hat{\boldsymbol{e}}_{3} \otimes \hat{\boldsymbol{e}}_{3} \\
& =\lambda_{2} \hat{\boldsymbol{e}}_{2} \otimes \hat{\boldsymbol{e}}_{2},
\end{aligned}
$$


where the base $\hat{\boldsymbol{e}}_{i}$ is an eigenvector of $\boldsymbol{\sigma}$, which can be determined by eigenvalue equation

$$
\boldsymbol{\sigma} \cdot \hat{\boldsymbol{e}}=2 k \hat{\boldsymbol{e}} \cdot \hat{\boldsymbol{e}}
$$

where the fluctuation kinetic energy $k=\frac{1}{2}\left[\left(u_{1}^{\prime}\right)^{2}+\left(u_{2}^{\prime}\right)^{2}+\right.$ $\left.\left(u_{3}^{\prime}\right)^{2}\right]$ results in:

$$
\hat{\boldsymbol{e}}=\hat{\boldsymbol{e}}_{2}=\frac{1}{\sqrt{2 k}} u_{\ell}^{\prime} \boldsymbol{e}_{\ell}=\frac{1}{\sqrt{2 k}} \boldsymbol{u}^{\prime} .
$$

Hence, we have

$$
\boldsymbol{\sigma}=\left[\left(u_{1}^{\prime}\right)^{2}+\left(u_{2}^{\prime}\right)^{2}+\left(u_{3}^{\prime}\right)^{2}\right] \hat{\boldsymbol{e}}_{2} \otimes \hat{\boldsymbol{e}}_{2},
$$

Therefore, the Reynolds tress tensor can also be

$$
\begin{aligned}
\boldsymbol{\tau} & =-\rho \lim _{T \rightarrow \infty} \frac{1}{T} \int_{t_{0}}^{t_{0}+T} \boldsymbol{\sigma} d t \\
& =-\rho\left(\lim _{T \rightarrow \infty} \frac{1}{T} \int_{t_{0}}^{t_{0}+T}\left[\left(u_{1}^{\prime}\right)^{2}+\left(u_{2}^{\prime}\right)^{2}+\left(u_{3}^{\prime}\right)^{2}\right] d t\right) \hat{\boldsymbol{e}}_{2} \otimes \hat{\boldsymbol{e}}_{2} .
\end{aligned}
$$

It is proven once again that both the tensor $\boldsymbol{\sigma}$ and the Reynolds stress tensor $\boldsymbol{\tau}$ are the only function of $u_{1}^{\prime}, u_{2}^{\prime}, u_{3}^{\prime}$. Summarily, the number of unknowns in the Reynolds stress tensor is shown in Table I below:

TABLE I: Number of unknowns in the Reynolds stress tensor

\begin{tabular}{c|c|c}
\hline & Current literature & This research study \\
\hline Number & 6 & 3 \\
\hline Unknowns & $\tau_{11}, \tau_{12}, \tau_{13}, \tau_{22}, \tau_{23}, \tau_{33}$ & $u_{1}^{\prime}, u_{2}^{\prime}$ and $u_{3}^{\prime}$ \\
\hline
\end{tabular}

Although the Reynolds-averaged Navier-Stokes (RANS) equations are unclosed, the four-equations RANS in Eqs. 5 and 6 contain only 7 unknowns instead of 10 . The list of unknowns in the RANS are summarized in the below Table II:

TABLE II: Number of unknowns in the RANS

\begin{tabular}{c|c|c}
\hline & Current literature & This research study \\
\hline Number & 10 & 7 \\
\hline Unknowns & $\bar{u}_{1}, \bar{u}_{2}, \bar{u}_{3}$ & $\bar{u}_{1}, \bar{u}_{2}, \bar{u}_{3}$ \\
& $\bar{p}$ & $\bar{p}$ \\
& $\tau_{11}, \tau_{12}, \tau_{13}, \tau_{22}, \tau_{23}, \tau_{33}$ & $u_{1}^{\prime}, u_{2}^{\prime}$ and $u_{3}^{\prime}$ \\
\hline
\end{tabular}

\section{TRANSPORT EQUATION OF REYNOLDS STRESS TENSOR}

In order to obtain more information about the Reynolds stress tensor, Reynolds [18] derived velocity fluctuation equations, namely the equation (16) in [18]), called the equations of momentum of relative-meanmotion at each point. Chou [30] reformulated these equations into index-tensorial at format. The equations are presented in below bold-face tensorial format as follows

$$
\begin{gathered}
\rho \boldsymbol{u}_{, t}^{\prime}+\rho \boldsymbol{\nabla} \cdot\left(\overline{\boldsymbol{u}} \otimes \boldsymbol{u}^{\prime}+\boldsymbol{u}^{\prime} \otimes \overline{\boldsymbol{u}}+\boldsymbol{u}^{\prime} \otimes \boldsymbol{u}^{\prime}\right)+\boldsymbol{\nabla} p^{\prime} \\
=\mu \boldsymbol{\nabla}^{2} \boldsymbol{u}^{\prime}+\rho \lim _{T \rightarrow \infty} \frac{1}{T} \int_{t}^{t+T} \boldsymbol{\nabla} \cdot\left(\boldsymbol{u}^{\prime} \otimes \boldsymbol{u}^{\prime}\right) d t, \\
\boldsymbol{\nabla} \cdot \boldsymbol{u}^{\prime}=0 .
\end{gathered}
$$

These are integral-differential equations governing $\overline{\boldsymbol{u}}, \boldsymbol{u}^{\prime}$ and $p^{\prime}$.

With the above new understanding of the number of unknowns, he following presents completely new perceptions on the higher-order correction of the Reynolds stress tensor.

Certain tensor algebra from Eqs.Eqs.36 and 37 resulted in an important equation for the Reynolds stress tensor, as follows

$$
\begin{aligned}
& \frac{\partial \boldsymbol{\tau}}{\partial t}+\overline{\boldsymbol{u}} \cdot \boldsymbol{\nabla} \boldsymbol{\tau}+\boldsymbol{\tau} \cdot \boldsymbol{\nabla} \overline{\boldsymbol{u}}+\boldsymbol{\nabla} \overline{\boldsymbol{u}} \cdot \boldsymbol{\tau} \\
& =2 \mu \boldsymbol{I}: \overline{\nabla \boldsymbol{u}^{\prime} \otimes \boldsymbol{\nabla} \boldsymbol{u}^{\prime}} \\
& +\overline{\boldsymbol{u}^{\prime} \otimes\left(\boldsymbol{\nabla} p^{\prime}\right)}+\overline{\left(\boldsymbol{\nabla} p^{\prime}\right) \otimes \boldsymbol{u}^{\prime}} \\
& +\mu \boldsymbol{\nabla}^{2} \boldsymbol{\tau}+\rho \boldsymbol{\nabla} \cdot \overline{\left(\boldsymbol{u}^{\prime} \otimes \boldsymbol{u}^{\prime} \otimes \boldsymbol{u}^{\prime}\right)}
\end{aligned}
$$

Current literature claims that Eq.38 has 31 unknowns. However, this research has a different opinion, and has proven that the Reynolds stress equation Eq. 38 only has 7 unknowns. Accoutring for all symmetries, these are listed in Table III below.

TABLE III: Number of unknowns in the Reynolds stress equation

\begin{tabular}{c|c|c}
\hline & Current literature & This research study \\
\hline Number & 31 & 7 \\
\hline Unknowns & $\overline{\boldsymbol{u}}(3)$ & $\bar{u}_{1}, \bar{u}_{2}, \bar{u}_{3}$ \\
& $\boldsymbol{u}^{\prime} \otimes \boldsymbol{u}^{\prime} \otimes \boldsymbol{u}^{\prime}(10)$ & $p^{\prime}$ \\
& $\boldsymbol{I}: \frac{\nabla \boldsymbol{u}^{\prime} \otimes \nabla \boldsymbol{u}^{\prime}}{\nabla}(6)$ & $u_{1}^{\prime}, u_{2}^{\prime}$ and $u_{3}^{\prime}$ \\
& $\frac{}{\boldsymbol{u}^{\prime} \otimes\left(\boldsymbol{\nabla} p^{\prime}\right)}(6)$ & \\
& $\boldsymbol{\tau}(6)$ & \\
\hline
\end{tabular}

The above statement is proven by the following:

$$
\begin{aligned}
& \boldsymbol{u}^{\prime} \otimes \boldsymbol{u}^{\prime} \otimes \boldsymbol{u}^{\prime}=\overline{u_{i}^{\prime} u_{j}^{\prime} u_{k}^{\prime}} \boldsymbol{e}_{i} \otimes \boldsymbol{e}_{j} \otimes \boldsymbol{e}_{k} \\
& =\left(\lim _{T \rightarrow \infty} \frac{1}{T} \int_{t_{0}}^{t_{0}+T} u_{i}^{\prime} u_{j}^{\prime} u_{k}^{\prime} d t\right) \boldsymbol{e}_{i} \otimes \boldsymbol{e}_{j} \otimes \boldsymbol{e}_{k},
\end{aligned}
$$

and

$$
\begin{aligned}
\boldsymbol{I} & : \overline{\nabla \boldsymbol{u}^{\prime} \otimes \boldsymbol{\nabla} \boldsymbol{u}^{\prime}}=\left(\boldsymbol{e}_{k} \otimes \boldsymbol{e}_{k}\right):\left(\overline{\boldsymbol{\nabla} \boldsymbol{u}^{\prime} \otimes \boldsymbol{\nabla} \boldsymbol{u}^{\prime}}\right) \\
& \left.=\overline{\left(\boldsymbol{e}_{k} \otimes \boldsymbol{e}_{k}\right):\left(\boldsymbol{\nabla} \boldsymbol{u}^{\prime} \otimes \boldsymbol{\nabla} \boldsymbol{u}^{\prime}\right.}\right) \\
& =\overline{\left(\boldsymbol{e}_{k} \cdot \boldsymbol{\nabla} \boldsymbol{u}^{\prime}\right) \otimes\left(\boldsymbol{e}_{k} \cdot \boldsymbol{\nabla} \boldsymbol{u}^{\prime}\right)} \\
& \left.\left.=\overline{\left(\boldsymbol{\nabla}_{k} \boldsymbol{u}^{\prime}\right) \otimes\left(\boldsymbol{\nabla}_{k} \boldsymbol{u}^{\prime}\right.}\right)=\overline{\left(\boldsymbol{\nabla}_{k} \tilde{u}_{i} \boldsymbol{e}_{i}\right) \otimes\left(\boldsymbol{\nabla}_{k} \tilde{u}_{j} \boldsymbol{e}_{j}\right.}\right) \\
& \left.=\overline{\left(\boldsymbol{\nabla}_{k} \tilde{u}_{i}\right)\left(\boldsymbol{\nabla}_{k} \tilde{u}_{j}\right.}\right) \boldsymbol{e}_{i} \otimes \boldsymbol{e}_{j}=\frac{\partial u_{i}^{\prime} \frac{\partial u_{j}^{\prime}}{\partial x_{k}}}{\partial x_{k}} \otimes \boldsymbol{e}_{j} \\
& =\left(\lim _{T \rightarrow \infty} \frac{1}{T} \int_{t_{0}}^{t_{0}+T} \frac{\partial u_{i}^{\prime}}{\partial x_{k}} \frac{\partial u_{j}^{\prime}}{\partial x_{k}} d t\right) \boldsymbol{e}_{i} \otimes \boldsymbol{e}_{j} .
\end{aligned}
$$


It is clear that the mean value of $\overline{u_{i}^{\prime} u_{j}^{\prime} u_{k}^{\prime}}$ and $\overline{\frac{\partial u_{i}^{\prime}}{\partial x_{k}} \frac{\partial u_{j}^{\prime}}{\partial x_{k}}}$ can be calculated by the velocity fluctuations $u_{1}^{\prime}, u_{2}^{\prime}$ and $u_{3}^{\prime}$. It means that $u_{1}^{\prime}, u_{2}^{\prime}$ and $u_{3}^{\prime}$ are unknowns. Similarly,

$$
\begin{aligned}
& \overline{\boldsymbol{u}^{\prime} \otimes\left(\boldsymbol{\nabla} p^{\prime}\right)}=\overline{u_{i}^{\prime} \frac{\partial p^{\prime}}{\partial x_{j}}} \boldsymbol{e}_{i} \otimes \boldsymbol{e}_{j} \\
& =\left(\lim _{T \rightarrow \infty} \frac{1}{T} \int_{t_{0}}^{t_{0}+T} u_{i}^{\prime} \frac{\partial p^{\prime}}{\partial x_{j}} d t\right) \boldsymbol{e}_{i} \otimes \boldsymbol{e}_{j}
\end{aligned}
$$

The mean value of $\overline{u_{i}^{\prime} \frac{\partial p^{\prime}}{\partial x_{j}}}$ can be calculated by $p^{\prime}$ and $u_{1}^{\prime}, u_{2}^{\prime}$ and $u_{3}^{\prime}$.

The same can also be done for the fourth order and for higher orders as in [30] and in textbooks. However, no other unknowns can be created by any order equation in respect of the Reynolds stress tensor.

\section{TRANSPORT EQUATION OF TURBULENCE KINETIC ENERGY}

The contraction operation for index $i$ and $j$ in Eq.38 resulted in the following transport equation for turbulence kinetic energy $k$

$$
\begin{aligned}
\rho\left(\frac{\partial k}{\partial t}+\overline{\boldsymbol{u}} \cdot \nabla k\right) & =\boldsymbol{\tau}: \boldsymbol{\nabla} \overline{\boldsymbol{u}} \\
& -\mu \overline{\boldsymbol{\nabla} \boldsymbol{u}^{\prime}: \boldsymbol{\nabla} \boldsymbol{u}^{\prime}}+\mu \boldsymbol{\nabla}^{2} k \\
& -\boldsymbol{\nabla} \cdot\left(\overline{p^{\prime} \boldsymbol{u}^{\prime}}\right)-\frac{1}{2} \rho \boldsymbol{\nabla} \cdot \overline{\left[\left(\boldsymbol{u}^{\prime} \cdot \boldsymbol{u}^{\prime}\right) \boldsymbol{u}^{\prime}\right]}
\end{aligned}
$$

where the kinetic energy $k=-\frac{1}{2} \tau_{k k}=\frac{1}{2} \overline{u_{k}^{\prime} u_{k}^{\prime}}=\frac{1}{2} \overline{\boldsymbol{u}^{\prime} \cdot \boldsymbol{u}^{\prime}}$.

\begin{tabular}{|c|c|c|}
\hline & Current literature & This research study \\
\hline Number & 21 & 7 \\
\hline Unknowns & $\begin{array}{c}k(3) \\
\overline{\boldsymbol{u}}(3) \\
\frac{\boldsymbol{\tau}(6)}{\nabla \boldsymbol{u}^{\prime}: \nabla \boldsymbol{u}^{\prime}}(3) \\
\frac{p^{\prime} \boldsymbol{u}^{\prime}}{\overline{\left[\left(\boldsymbol{u}^{\prime} \cdot \boldsymbol{u}^{\prime}\right) \boldsymbol{u}^{\prime}\right]}}\end{array}$ & $\begin{array}{c}u_{1}^{\prime}, u_{2}^{\prime} \text { and } u_{3}^{\prime} \\
\bar{u}_{1}, \bar{u}_{2}, \bar{u}_{3} \\
p^{\prime}\end{array}$ \\
\hline
\end{tabular}
The number of unknowns in the kinetic energy equation Eq.42 is listed in Table IV below.

TABLE IV: Number of unknowns in the kinetic energy equation

\section{GENERAL COMPUTATIONAL ALGORITHM OF TURBULENT FLOW}

Within Reynolds original framework the following algorithm is proposed for three dimensional turbulent flow:

(1) Find a solution for corresponding equations of viscous steady flow $\overline{\boldsymbol{u}}$ :

$$
\nabla \cdot(\overline{\boldsymbol{u}} \otimes \overline{\boldsymbol{u}})=\nu \nabla^{2} \overline{\boldsymbol{u}} .
$$

(2) Calculate the pressure (source term) $\bar{p}$ :

$$
\nabla^{2} \bar{p}=-\rho \nabla \cdot(\overline{\boldsymbol{u}} \cdot \boldsymbol{\nabla} \overline{\boldsymbol{u}}) .
$$

(3) Propose an approximate solution for velocity fluctuations $\boldsymbol{u}^{\prime}$ :

$$
\boldsymbol{u}^{\prime}=\sqrt{2} \boldsymbol{C}(\boldsymbol{x}) \cos (\omega t)
$$

(4) Calculate the Reynolds stress tensor $\boldsymbol{\tau}$ :

$$
\boldsymbol{\tau}=-\rho \lim _{T \rightarrow \infty} \frac{1}{T} \int_{t}^{t+T}\left(\boldsymbol{u}^{\prime} \otimes \boldsymbol{u}^{\prime}\right) d t=-\rho(\boldsymbol{C} \otimes \boldsymbol{C}) .
$$

(5) Determine the coefficient vector $\boldsymbol{C}$ : Substitute $\overline{\boldsymbol{u}}, \bar{p}, \boldsymbol{\tau}$ into the RANS, hence we have

$$
\boldsymbol{\nabla} \cdot(\overline{\boldsymbol{u}} \otimes \overline{\boldsymbol{u}})-\nu \nabla^{2} \overline{\boldsymbol{u}}=-\frac{1}{\rho} \nabla \bar{p}-\nabla \cdot(\boldsymbol{C} \otimes \boldsymbol{C}) .
$$

Since $\overline{\boldsymbol{u}}$ is the solution for $\boldsymbol{\nabla} \cdot(\overline{\boldsymbol{u}} \otimes \overline{\boldsymbol{u}})=\nu \nabla^{2} \overline{\boldsymbol{u}}$, therefore, the left side of the above equation is zero, hence

$$
\boldsymbol{\nabla} \cdot\left(\boldsymbol{C} \otimes \boldsymbol{C}+\frac{1}{\rho} \bar{p} \boldsymbol{I}\right)=0
$$

This relation reveals that the fluctuation magnitude is proportional to pressure, the turbulence can not maintained without source supplying.

(6) Calculate the pressure fluctuation $p^{\prime}$

$$
\nabla^{2} p^{\prime}=-\rho \nabla \cdot\left[\bar{u} \cdot \nabla \boldsymbol{u}^{\prime}+\boldsymbol{u}^{\prime} \cdot \nabla \bar{u}+\boldsymbol{u}^{\prime} \cdot \nabla \boldsymbol{u}^{\prime}\right]-\tau .
$$

(7) Calculate the total pressure $p$ :

$$
p=\bar{p}+p^{\prime}
$$

(8) Calculate the total velocity field $\boldsymbol{u}$

$$
\boldsymbol{u}=\overline{\boldsymbol{u}}+\boldsymbol{C}(\boldsymbol{x}) \cos \omega t
$$

Similarly, one can obtain higher order solutions.

\section{EXAMPLES OF FINDING TURBULENCE SOLUTION}

The following two examples shed some light on the above number of unknowns. The aim of these exercises are for demonstration purposes: to show algorithm to find turbulence solutions. The study has not provided complete solutions here as its key focus was to determine the number of unknowns in the Reynolds-averaged Navier-Stokes equations. The comprehensive study and detailed numerical simulations of those examples might be presented in the future. 


\section{A. Burgers equation}

This example will show that the Reynolds decomposition is a method to solve nonlinear problem. The one dimensional Navier-Stokes equation, namely the Burgers equation [63]

$$
u_{, t}+u u_{, x}=\nu u_{, x x}+S(x, t),
$$

where the source term $S(x, t)$ is

$$
\begin{aligned}
S(x, t) & =\cos (x+t)+\nu \sin (x+t) \\
& +B \cos (x+t) \tanh \left(A-\frac{B}{2 \nu} x\right) \\
& +\sin (x+t) \cos (x+t) \\
& -\frac{1}{2 \nu} B^{2} \sin (x+t)\left[1-\tanh ^{2}\left(A-\frac{1}{2 \nu} B x\right)\right],
\end{aligned}
$$

where the trigonometric tangent function is defined as $\tanh (x)=\frac{\sinh (x)}{\cosh (x)}=\frac{e^{x}-e^{-x}}{e^{x}+e^{-x}}$, and $A, B$ are constant.

Substituting the Reynolds velocity decomposition $u=$ $\bar{u}(x)+u^{\prime}(x, t)$ into Eq.52, after time-averaging, results in its RANS as follows

$$
\bar{u} \bar{u}_{, x}+\overline{u^{\prime} u_{, x}^{\prime}}=\nu \bar{u}_{, x x}+\bar{S},
$$

namely

$$
\bar{u} \bar{u}_{, x}+\lim _{T \rightarrow \infty} \frac{1}{T} \int_{t_{0}}^{t_{0}+T} u^{\prime} u_{, x}^{\prime} d t=\nu \bar{u}_{, x x}+\bar{S},
$$

where the mean source term $\bar{S}=\frac{1}{2}$. The Eq. 55 only has two unknowns; one is the mean velocity $\bar{u}$ and another is the velocity fluctuation $u^{\prime}$, as stated previously part.

Since we only have one integral-differential equation Eq.55, therefore, Eq.55 is not closed. To solve Eq.55, we have to model either the mean field $\bar{u}$ or fluctuation $u^{\prime}$.

We can use the steady solution of $\bar{u} \bar{u}_{, x}=\nu \bar{u}_{, x x}$ as the 1st order approximate solution of the mean field. Hence, the 1st order exact solution is:

$$
\bar{u}(x)=B_{1} \tanh \left(A_{1}-\frac{B_{1}}{2 \nu} x\right),
$$

where the constants $A_{1}, B_{1}$ will be determined later.

For fluctuation field $u^{\prime}$, since its mean $u^{\prime}$ must be zero, namely $\lim _{T \rightarrow \infty} \frac{1}{T} \int_{t_{0}}^{t_{0}+T} u^{\prime} d t \equiv 0$, we can accept the 1 st order velocity fluctuation, proposed by Reynolds [18], as an approximate solution

$$
u^{\prime}(x, t)=\sqrt{2} a \sin (\omega t+\kappa x)
$$

where $a$, frequency $\omega$ and wave number $\kappa$ should be determined.

Substituting both Eqs.56 and 57 into Eq.55, results in

$$
a=\frac{1}{\sqrt{2 \kappa}} .
$$

Therefore, we have the 1 st order velocity field is as follows

$$
u(x, t)=B_{1} \tanh \left(A_{1}-\frac{B_{1}}{2 \nu} x\right)+\frac{1}{\sqrt{2 \kappa}} \sin (\omega t+\kappa x) .
$$

Initial-boundary conditions $u(0,0)=1, u_{, x}(0,0)=1$, result in $B_{1}=\sqrt{1+\nu}, \tanh \left(A_{1}\right)=\frac{1}{\sqrt{1+\nu}} u_{, t}(0,0)=$ $1, u_{, x x}(0,0)=1$, and $\omega=\sqrt{\kappa}$ and $\kappa=\frac{1+\nu+\sqrt{1+\nu}}{2 \nu^{2}}-1$.

Hence, the 1st order solution is

$$
\begin{aligned}
u(x, t) & =\sqrt{1+\nu} \tanh \left(A_{1}-\frac{\sqrt{1+\nu}}{2 \nu} x\right) \\
& +\frac{1}{\sqrt{2 \kappa}} \sin (\sqrt{\kappa} t+\kappa x) .
\end{aligned}
$$

(1) The 1 st order turbulence velocity $u_{1 s t}$ is different to the exact solution of the Burgers equation in Eq.52. The exact solution of Burgers equation is

$$
u_{\text {exact }}(x, t)=B \tanh \left(A-\frac{B}{2 \nu} x\right)+\sin (x+t) .
$$

(2) In particular, when $x \rightarrow \infty$, their asymptotic behavior is completely different. The exact solution will be $u_{\text {exact }}=B+\sin (x+t)$ owing to the property of $\lim _{x \rightarrow \infty} \tanh (x) \rightarrow 1$, while the turbulence solution

$$
u(x, t)=\sqrt{1+\nu}+\frac{1}{\sqrt{2 \kappa}} \sin (\sqrt{\kappa} t+\kappa x) . .
$$

(3) The turbulence kinetic energy is $k=1 /(2 \kappa)$.

\section{B. Unsteady turbulent boundary layer for incompressible two-dimensional flow}

Here the velocity components $u=u_{1}, v=u_{2}$, directions $1 \rightarrow x, 2 \rightarrow y$ are adopted respectively.

According to Prandtl's boundary layer concept [24, 62], the two dimensional Navier-Stokes equations can be simplified to following boundary layer equations:

$$
\begin{aligned}
u_{, t}+u u_{, x}+v u_{, y} & =-\frac{1}{\rho} \frac{\partial p}{\partial x}+\mu u_{, y y}, \\
u_{, x}+v_{, y} & =0
\end{aligned}
$$

and boundary conditions:

$$
y=0: u=0, v=0 .
$$

In duct and boundary layer flow, the following are simpler turbulent boundary layer equations [50]:

$$
\begin{aligned}
\rho\left(\bar{u} \bar{u}_{, x}+\bar{v} \bar{u}_{, y}\right) & =-\frac{1}{\rho} \frac{\partial p}{\partial x}+\mu \bar{u}_{, y y}+\tau_{x x, x}+\tau_{x y, y}, \\
\bar{u}_{, x}+\bar{v}_{, y} & =0
\end{aligned}
$$

and boundary conditions:

$$
y=0: \bar{u}=0, \bar{v}=0,
$$


where turbulence Reynolds stresses are

$$
\begin{aligned}
& \tau_{x x}=-\rho \overline{u^{\prime} u^{\prime}}=-\rho \lim _{T \rightarrow \infty} \frac{1}{T} \int_{t_{0}}^{t_{0}+T} u^{\prime} u^{\prime} d t \\
& \tau_{x y}=-\rho \overline{u^{\prime} v^{\prime}}=-\rho \lim _{T \rightarrow \infty} \frac{1}{T} \int_{t_{0}}^{t_{0}+T} u^{\prime} v^{\prime} d t .
\end{aligned}
$$

Noting $\tau_{x x}$ is omitted in the Plandtl's original boundary layer equations, we keep the term to prevent the zero turbulence scenario, which will be shown later.

To solve the above equations, we firstly omit the fluctuation term and reduce Eqs.66 and 67 into the followings

$$
\begin{aligned}
\rho\left(\bar{u} \bar{u}_{, x}+\bar{v} \bar{u}_{, y}\right) & =\mu \bar{u}_{, y y}, \\
\bar{u}_{, x}+\bar{v}_{, y} & =0,
\end{aligned}
$$

Blasius [62] obtained their solutions as follows

$$
\begin{aligned}
& \bar{u}=U f^{\prime}(\xi), \\
& \bar{v}=\frac{1}{2} \sqrt{\frac{\nu U}{x}}\left(\xi f^{\prime}-f\right),
\end{aligned}
$$

where $\xi=y \sqrt{\frac{U}{\nu x}}, U=u_{e}(0), f^{\prime}=\frac{d f}{d \xi}$, and function $f(\xi)$ is the solution for equation $f f^{\prime \prime}+2 f^{\prime \prime \prime}=0$ under conditions $f(0)=f^{\prime}(0)=0, f^{\prime}(\infty)=1$.

For fluctuation fields $u^{\prime}$ and $v^{\prime}$, since their mean must be vanish, we can take the 1st order velocity fluctuation, as suggested by Reynolds [18], as their solutions

$$
\begin{aligned}
& u^{\prime}=\sqrt{2} c_{1}(x, y) \cos (\omega t), \\
& v^{\prime}=\sqrt{2} c_{2}(x, y) \cos (\omega t),
\end{aligned}
$$

where round frequency $\omega$ is determined by an initial condition.

Substituting Eqs. 73,74,75 and 76 into Eq. 66, we obtain

$$
\left(c_{1}^{2}\right)_{, x}+\left(c_{1} c_{2}\right)_{, y}=-\frac{1}{\rho} \frac{\partial \bar{p}}{\partial x} .
$$

To determine $c_{1}, c_{2}$, we need the mass conversation law of the fluctuation field $u_{, x}^{\prime}+v_{, y}^{\prime}=0$, namely $c_{1, x}+c_{2, y}=$ 0 . To find their special solutions, Eq.77 and the mass conversation can be reformulated as follows

$$
\begin{aligned}
-\frac{1}{2 x} c_{1, \xi}+\frac{1}{y} c_{2, \xi} & =0, \\
-\frac{1}{2 x}\left(c_{1}^{2}\right)_{, \xi}+\frac{1}{y}\left(c_{1} c_{2}\right)_{, \xi} & =\frac{1}{2 \rho} \frac{1}{x} \bar{p}_{, \xi} .
\end{aligned}
$$

Combining the above relations results in

$$
\left(c_{1} c_{2}\right)_{, \xi}-2 c_{1} c_{2, \xi}=\frac{1}{2 \rho} \frac{\xi}{\sqrt{R e_{x}}} \bar{p}_{, \xi} .
$$

where the Reynolds number $R e_{x}=\frac{U x}{\nu}$ in $x$ direction.

It is obvious that the final solutions of $c_{1}, c_{2}$ depends on the flow pressure $\bar{p}$.
(1) If omit $\tau_{x x}$ as in Plantdl boundary layer equations, namely $c_{1}=0$, then $c_{2}=0$, it means that there is no turbulence.

(2) Constant pressure $\bar{p}$ leads to $\bar{p}_{, \xi}=0$, hence $c_{1}=$ $-c_{2}=C$, where $C$ is a constant.

(3) For a large Reynolds number $R e_{x} \rightarrow \infty$, the right side of Eq.80 tends to null, this gives $c_{1}=-c_{2}=C$ as well. Hence, the velocity fluctuation are obtained as follows

$$
\begin{aligned}
& u^{\prime}=\sqrt{2} C \cos (\omega t), \\
& v^{\prime}=-\sqrt{2} C \cos (\omega t),
\end{aligned}
$$

and the first order total velocity field can be written as follows

$$
\begin{aligned}
& u=U f^{\prime}(\xi)+\sqrt{2} C \cos (\omega t), \\
& v=\frac{1}{2} \sqrt{\frac{\nu U}{x}}\left(\xi f^{\prime}-f\right)-\sqrt{2} C \cos (\omega t),
\end{aligned}
$$

and the Reynolds stresses are given by

$$
\begin{aligned}
& \tau_{x x}=-\rho \overline{u^{\prime} u^{\prime}}=-\rho C^{2}, \\
& \tau_{x y}=-\rho \overline{u^{\prime} v^{\prime}}=\rho C^{2} .
\end{aligned}
$$

The magnitude of $\tau_{x x}$ is same as $\tau_{x y}$, that is why we keep the $\tau_{x x}$ in boundary formulation. The above solutions reveal that the turbulence character will stationary for both large Reynolds number and constant pressure.

(4) If flow pressure is $\bar{p}=-60 \rho\left(\frac{U x}{\nu}\right) \xi^{4}$, then we have

$$
\begin{aligned}
& c_{1}(x, y)=-\left(\frac{U}{\nu}\right)^{3 / 2} x^{-1 / 2} y^{2}=-\xi^{2} \sqrt{\frac{U x}{\nu}}, \\
& c_{2}(x, y)=\frac{1}{6}\left(\frac{U}{\nu}\right)^{3 / 2} x^{-3 / 2} y^{3}=\frac{1}{6} \xi^{3}
\end{aligned}
$$

Hence, the velocity fluctuations are obtained as follows

$$
\begin{aligned}
& u^{\prime}=-\sqrt{2} \xi^{2} \sqrt{\frac{U x}{\nu}} \cos (\omega t), \\
& v^{\prime}=\frac{\sqrt{2}}{6} \xi^{3} \cos (\omega t),
\end{aligned}
$$

and the first order total velocity field can be written as follows

$$
\begin{aligned}
& u=U f^{\prime}(\xi)-\sqrt{2 R e_{x}} \xi^{2} \cos (\omega t), \\
& v=\frac{1}{2} \sqrt{\frac{\nu U}{x}}\left(\xi f^{\prime}-f\right)+\frac{\sqrt{2}}{6} \xi^{3} \cos (\omega t),
\end{aligned}
$$

and the Reynolds stresses are given by

$$
\begin{aligned}
& \tau_{x x}=-\rho \overline{u^{\prime} u^{\prime}}=-\rho R e_{x} \xi^{4}, \\
& \tau_{x y}=-\rho \overline{u^{\prime} v^{\prime}}=\rho \sqrt{\operatorname{Re}_{x}} \xi^{5},
\end{aligned}
$$

where frequency $\omega$ and constant $\mathrm{C}$ are determined by initial conditions. The solution in case 3 reveals that the turbulence character will change at different position 


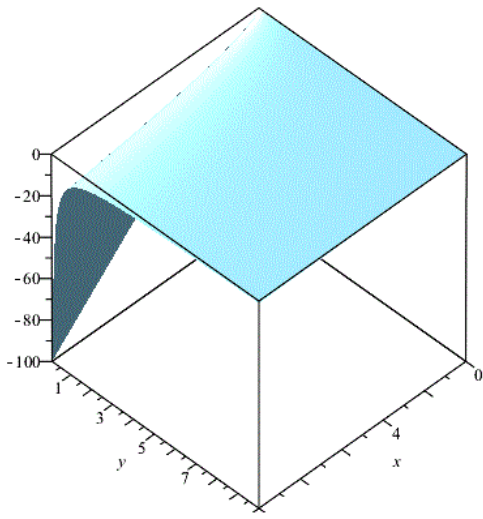

FIG. 1: The ratio of Reynolds stress: $\tau_{x x} / \tau_{x y}=-x / y$. The $\tau_{x x} \sim \tau_{x y}$, when $(x, y) \rightarrow(\infty, \infty)$; for a fixed $x$, if $y$ is smaller than 1 , namely $y \ll 1$, near the boundary the ratio could be quite large, namely $\tau_{x x} \gg \tau_{x y}$; therefore $\tau_{x x}$ cannot be omitted in boundary layer formulations.

$x, y$. The ratio $\frac{\tau_{x x}}{\tau_{x y}}=-\frac{x}{y}$ is plotted in Figure 1 . It is worth mentioning that the above solutions have never been apparent in the literature.

We must point out here that the one and/or two dimensional Navier-Stokes model can not represent a real turbulence, since turbulence motion is always three dimensional [8].

\section{CONCLUSIONS}

This article re-visited a fundamental problem in turbulence analysis, namely the number of unknowns in the Reynolds stress tensor. The research study found that there are 3 unknowns in the Reynolds stress tensor, namely 3 components of velocity fluctuations $u_{1}^{\prime}, u_{2}^{\prime}$ and $u_{3}^{\prime}$. This study has not only clarified the number of unknowns in the formulations of the Reynolds- averaged Navier-Stokes equation, but has also discovered that the number of unknowns is much less than traditionally found and thought.

For application purposes, this study has resolved the Burgers equation and the turbulent Prandtl boundary layer equations. None of the solutions have any adjustable parameters. The study found that the Reynolds stress depends mainly on the source term (flow pressure), which means that the turbulence cannot be maintained if there is no steady source.

In future, turbulence modelling should focus on the modelling of velocity fluctuations $u_{1}^{\prime}, u_{2}^{\prime}, u_{3}^{\prime}$, instead of on the Reynolds stress $\tau_{i j}$. An advantage of modelling the velocity fluctuations $u_{1}^{\prime}, u_{2}^{\prime}, u_{3}^{\prime}$ is that it reduces the 6 components of $\tau_{i j}$ into 3 , and from an experimental perspective, the components of velocity fluctuations are easiest to measure than the Reynolds stress tensor.

It is important to point out that this study's ideas and methodologies are also applicable to compressible turbulence Navier-Stokes equations, where the mass density $\rho$ and the temperature $T$ must be taken into account, and their Reynolds decompositions, $\rho=\bar{\rho}+\tilde{\rho}$ and $T=\bar{T}+\tilde{T}$, should be introduced.

The present investigation can be considered as a renaissance of Reynolds' study in 1895, which might assist with understanding the well-known closure problem of turbulence, and should help to understand the puzzle of the turbulence closure problem that has eluded scientists and mathematicians for centuries.

\section{ACKNOWLEDGEMENT}

It is my great pleasure to have shared and discussed some thoughts of this paper with Michael Sun of Bishops Diocesan College, whose pure and direct scientific knowledge inspired me. This paper is dedicated to the memory of my beloved father, Zhong-Chuan Sun.
[1] https://en.wikipedia.org/wiki/Turbulence

[2] https://ideasranking.com/turbulence-one-of-the-greatunsolved-mysteries-of-physics-ted-ed.html

[3] D. Castelvecchi, Mysteries of turbulence unravelled, Nature, 548:382 (2017).

[4] K.R. Sreenivasan, On the scaling of the turbulence energy dissipation rate. Physics of Fluids, 27,5:1048-1051 (1984).

[5] U. Frisch, Turbulence: The Legacy of A.N. Kolmogorov. Cambridge University Press, Cambridge (2008).

[6] G.K. Batchelor, The Theory of Homogeneous Turbulence. Cambridge University Press (1953).

[7] P. Bradshaw, An Introduction to Turbulence and Its Measurement. Pergamon Press, New York (1971).

[8] H. Tennekes and J.L. Lumley, A First Course in Turbulence. Cambridge: The MIT Press (1972).

[9] D.C. Lesilie, Developments in the Theory of Turbulence.
Clarendon Press, Oxford (1973).

[10] A.A. Townsend, The Structure of Turbulent Shear Flow 2nd ed., Cambridge University Press, New York (1976).

[11] M. Lesieur, Turbulence in Fluids. 2nd ed. Kluwer, Dordrecht (1990).

[12] D.C. Wilcox, Turbulence Modeling for CFD. D C W Industries (1993).

[13] S.B. Pope, Turbulent Flows. Cambridge University Press, Cambridge (2000).

[14] P.A. Davidson, Turbulence. Oxford University Press, Oxford (2004).

[15] B. Hof, Experimental Observation of Nonlinear Traveling Waves in Turbulent Pipe Flow. Science 305, 1594 (2004).

[16] G. Falkovich and K.R. Sreenivasan, Lessons from hydrodynamic turbulence. Physics Today, 43-49 (April 2006).

[17] P.A. Davison, et al. A Voyage Through Turbulence. Cambridge: Cambridge University Press (2011). 
[18] O. Reynolds, On the dynamical theory of incompressible viscous fluids and the determination of the criterion. Philos. Trans. R. Soc. 186:123-164(1895).

[19] Sir H. Lamb, Hydrodynamics, 6th edition, Cambdridge University Press, 1993.

[20] C. L. M. H. Navier, Mémoire sur les Lois du Mouvement des Fluides, Mém.de l'Acad.des Sciences, vi.389 (1822).

[21] S.D. Poisson, Mémoire sur les Équations géérales de l'Équilibre et du Mouvement des Corps solides élastiques et des Fluides, Journ.de l'École Plytechn.xiii. 1 (1829).

[22] M. B. de Saint-Venant, Comptes Rendus, xvii. 1240(1843).

[23] G.G. Stokes, On the Theories of the Internal Friction of Fluids in Motion and of the Equilibrium and Motion of Elastic Solids. Trans. Cambridge Philos. Soc., 8, 287319(1845).

[24] L. Prandtl, On fluid motions with very small friction (in German). Third International Mathematical Congress, Heidelberg. 484-491(1904).

[25] W. Heisenburg, On stability and turbulence of fluid flow, Annalen der Physik. Vol. 74, No. 15, pp. 577-627, 1924.

[26] L. F. Richardson, Weather Prediction by Numerical Process. Cambridge University Press, 1922.

[27] L. Prandtl, Bericht uber die entstehung der turbulenz. Z. Angew. Math. Mech 5, 136(1925).

[28] G. I. Taylor. Statistical theory of turbulence. Proc. R. Soc. Lond. A, 151:421-444, 1935.

[29] T. von Karman and L. Howarth On the statistical theory of isotropic turbulence. Proc. R. Soc. Lond. A, 164:192215,1938

[30] P-Y. Chou, On velocity correlations and the solutions of the equations of turbulent fluctuation. Q. Appl. Math. 111(1):38-54(1945).

[31] P-Y. Chou and R.L. Chou, 50 years of turbulence research in China. Annu. Rev. Fluid Mech. 27:1-15(1995).

[32] R. H. Kraichnan, Hydrodynamic turbulence and the renormalization group. Phys.Rev. A, 25:3281-3289, 1982.

[33] S.Y. Chen and P.-Y. Zhou, The application of quasisimilarity conditions in turbulence modeling theory. Journal of Hydrodynamics, 2(2) (1987)

[34] P. R. Spalart and S. R. Allmaras. A one-equation turbulence model for aerodynamics flows. La Recherche Aerospatiale, 1:5-21, 1994.

[35] P. Moin and K. Mahesh. Direct numerical simulation: a tool in turbulence research. Annu. Rev. Fluid Mech., 30:539-578, 1998.

[36] C.B. Lee and J.Z. Wu, Transition in wall-bounded flows. Applied Mechanics Reviews, 61(3), 030802 (2008).

[37] I. Marusic, R. Mathis and N. Hutchins, Predictive model for wall-bounded turbulent Flow. Science 329, 193 (2010).

[38] A.J. Smits, B.J. McKeon and I. Marusic, High-Reynolds Number Wall Turbulence, Annu. Rev. Fluid Mech. 43, $353(2011)$.

[39] B. Suri, J.R. Tithof, R.O. Grigoriev and M.F. Schatz, Forecasting Fluid Flows Using the Geometry of Turbulence. Phys. Rev. Lett. 118, 114501 (2017).

[40] D. Castelvecchi, On the trial of turbulence. Nature, 548:382 (2017).

[41] L.D. Landau and E. M. Lifshitz, Mechanics (3rd ed.) (Butterworth-Heinemann, Oxford, 1976).

[42] A.N. Kolmogorov, The local structure of turbulence in incompressible viscous fluid for very large Reynolds number. Dokl. Akad. Nauk SSSR, 30:299-303 (1941a) (reprinted in Proc.R.Soc.Lond. A, 434,9-13, 1991).

[43] A.N. Kolmogorov, On degeneration (decay) of isotropic turbulence in an incompressible visous liquid. Dokl. Akad. Nauk SSSR, 31:538-540 (1941b).

[44] A.N. Kolmogorov, Dissipation of energy in locally isotropic turbulence. Dokl.Akad. Nauk SSSR, 32:1618 (1941c).(reprinted in Proc.R.Soc.Lond. A, 434,15-17, 1991).

[45] N. Cao, S. Chen, and Z. S. She, Scalings and relative scalings in the Navier-Stokes turbulence. Phys. Rev. Lett., 76:3711-3714, 1996.

[46] Z.S. She and E. Lévêque, Universal scaling laws in fully developed turbulence. Phys. Rev. Lett. 72,336(1994).

[47] E.N. Lorenz, Deterministic non-periodic flow. J. Atmos Sci. 20:130-41(1963).

[48] R. Benzi, P. Paladin, G. Parisis and A. Vulpiani, On the multifractal nature of fully developed turbulence and chaotic systems. J. Phys. A: Math. Gen. 17:35213531(1984).

[49] S.A. Orszag and G.S. Patterson, Numerical simulation of three-dimensional homogeneous isotropic turbulence. Phys. Rev. Lett. 28,76(1972).

[50] H. Schlichting and K. Gersten, Boundary Layer Theory, 8th edition, Springer (2000).

[51] B. Sun, The temporal scaling laws of compressible turbulence. Modern Physics Letters B. 30,(23) 1650297 (2016).

[52] B. Sun, Scaling laws of compressible turbulence. Appl. Math. Mech.-Engl. Ed. 38: 765(2017).

[53] B. Sun, Thirty years of turbulence study in China. Applied Mathematics and Mechanics (English Edition), 40(2), 193-214 (2019); https://doi.org/10.1007/s10483$019-2427-9$

[54] B. Sun, A additive decomposition of velocity gradient, Physics of Fluids 31, 061702(2019); doi:10.1063/1.5100872.

[55] B. Sun, On the Reynolds-averaged Navier-Stokes equations. Preprints 2019, doi:10.20944/preprints201907.0038.v1.

[56] B. Sun, On closure problem of incompressible turbulent flow. Preprints 2018, 2018070622, doi: 10.20944/preprints201807.0622.v2.

[57] B. Sun, An Intrinsic Formulation of Incompressible Navier-Stokes Turbulent Flow. Preprints 2018, 2018080071, doi: 10.20944/preprints201808.0071.v3.

[58] B. Sun, A Novel Simplification of the ReynoldsChou-Navier-Stokes Turbulence Equations of Incompressible Flow. Preprints 2018, 2018070030, doi: 10.20944/preprints201807.0030.v1.

[59] B. Sun and Elaine S. Oran, New principle for aerodynamic heating, National Science Review 00: 1-2, 2018, doi: $10.1093 / \mathrm{nsr} / \mathrm{nwy} 035$.

[60] H. Hu, and B. Sun, New development in near-wall PIV measurements, Sci. China-Phys. Mech. Astron. 61, 094731 (2018)

[61] https://en.wikipedia.org/wiki/Dyadic_tensor

[62] H. Blasius, Grenzschichten in Fljssigkeiten mit kleiner Reibung. Z. Math.Phys., 56 1-37(1908).

[63] J. M. Burgers. A mathematical model illustrating the theory of turbulence. Advances in Applied Mechanics, Vol. I, 171-199, Academic, New York, 1948. 